\title{
A New Wireless VoIP Signaling Device Supporting SIP and H.323 Protocols
}

\author{
Salma Rattal, ${ }^{1}$ Abdelmajid Badri, ${ }^{1}$ and Mohammed Moughit ${ }^{2}$ \\ ${ }^{1}$ Faculty of Sciences and Techniques, Hassan 2nd University Mohammedia-Casablanca, Casablanca, Morocco \\ ${ }^{2}$ National School of Applied Sciences, Hassan 1st University, Khouribga, Morocco
}

Correspondence should be addressed to Salma Rattal; rattal.salma@gmail.com

Received 19 March 2014; Accepted 25 April 2014; Published 2 July 2014

Academic Editor: Tzonelih Hwang

Copyright (C) 2014 Salma Rattal et al. This is an open access article distributed under the Creative Commons Attribution License, which permits unrestricted use, distribution, and reproduction in any medium, provided the original work is properly cited.

\begin{abstract}
Nowadays, VoIP is a technology with a great demand and wireless networks are increasingly deployed. Each of these has its own technology constraints. For VoIP, it is very important to take into consideration the need to provide a high quality service according to well-defined standard transmission (jitter, end-to-end delay, MOS, and packet loss). However, wireless networks (IEEE 802.11) are based on radio which undergoes a number of technical constraints to achieve theoretical transmission rates; among these constraints the number of users of the networks, the distance between the client and the access, and the amount of data transmitted point are included. In this term, a study is made by simulating wireless network in OPNET Modeler with a fairly large number of VoIPs (15 users) whose signaling is handled via a new node that was created specifically to manage the signaling tasks under SIP and H.323 in order to minimize the number of nodes in the network and avoid the congestion. In this paper, two scenarios are compared; the first contains a number of VoIP users with SIP and H.323 signaling handled by the new created device; the second scenario is similar to the first except that the distance between the stations is remarkably lower.
\end{abstract}

\section{Introduction}

VoIP has evolved rapidly with a huge success in recent years and the current WLAN infrastructure on the market has lacked the scalability, quality, and usability needed to support large-scale deployments. The two technologies together have given rise to an exciting application: wireless VoIP.

However, conversational multimedia over wireless faces some challenges due to the inherent properties of the wireless access network. The requirements of low jitter, of consistent and low end-to-end delay, and of limited packet loss essential to good quality-service and intelligible VoIP communications are difficult to achieve in a time-varying environment due to channel errors and traffic congestion [1].

Wireless VoIP needs to use a large number of protocols and techniques. Signaling is an important part of call establishment and the most common signaling protocols are SIP, proposed by Internet Engineering Task Force (IETF) as a standard of IP telephony [2] but it is not the only possible choice; IUT-T H.323 is an alternative protocol which imposes different topology to accomplish the same tasks as his competitor.

In OPNET Modeler, proxies and gatekeepers are available to establish SIP or H.323 scenarios separately. But in this paper, a new element capable of managing heterogeneous SIP/H.323 communications in the same wireless network (IEEE 802.11) is presented. With the new tools created, it has become possible to simulate scenarios including both wireless SIP and wireless H.323 together with performance evaluation of this new equipment.

This paper tackles the following points; the first section will be devoted to study the wireless local area network (WLAN) standardized under IEEE 802.11 family. In the second section, a comparison is drawn between SIP and H.323 and the way that they operate into a VoIP network. Then, the third section includes the main work of that paper: it concerns the way of modeling a wireless SIP Proxy prototype with the integration of H.323 protocol in OPNET Modeler (project, node model, and process model). Finally, discussion and a conclusion summarize the results of this work. 


\section{IEEE 802.11 Local Area Network}

Nowadays, the IEEE 802.11 WLAN technology offers the largest deployed wireless access to the Internet. This technology specifies both the medium access control (MAC) and the physical layers (PHY) [3].

2.1. Mac Layer Protocols. The MAC layer is responsible for the channel allocation procedures, the protocol data unit addressing, the frame formatting, the error checking, the fragmentation, and the reassembly. The medium can alternate between contention period and contention free period. In contention mode, all stations are required to contend for access to the channel for each packet transmitted. During the contention free period, the access point controls the medium and assigns access times to the stations.

IEEE 802.11 supports three coordination function schemes. Distributed coordination function (DCF) is based on using carrier-sensing multiple access with collision avoidance (CSMA/CA). There are numerous CSMA protocols and their performances under low load conditions are usually similar $[4,5]$. The many variations arise because of efforts to improve on performance and push back the limits.

DCF with handshaking RTS/CTS procedure where a station wanting to transmit a packet transmits first a short control packet called RTS; if the medium is free, the destination station sends back a response control packet called CTS.

Point coordination function (PCF) involves a point coordinator (e.g., access point) that has priority control of the medium. The point coordinator performs poll-and-response protocol to allow the polled stations to transmit without contending for the channel [1].

2.2. Physical Layer. The 802.11 standard family includes various physical layer specifications defined in a number of amendments: 802.11a, 802.11b, 802.11g, and 802.11n.

As far as the physical layer of the "original" IEEE 802.11 [6] is concerned, three different basic transmission techniques offering 1 and $2 \mathrm{Mbps}$ data rate are defined as follows [6].

(i) Diffuse infrared (IR).

(ii) GHz ISM band frequency-hopping spread-spectrum (FHSS) which implies the change of carrier frequency after a short while.

(iii) $2.4 \mathrm{GHz}$ ISM band direct sequence spread spectrum (DSSS) which consists of spreading the transmit signal by multiplying it with a signal having a much larger bandwidth.

For the IEEE 802.11b PHY, a high rate mode extension has been introduced enabling 5.5 and $11 \mathrm{Mbps}$. The physical layer uses the complementary code keying as its modulation scheme that defines a subset of codewords as valid codewords based on the complementary property of the code sequences. Two amendments IEEE 802.11a and IEEE 802.11g have been developed providing up to $54 \mathrm{Mbps}$, respectively, in the $5 \mathrm{GHz}$ unlicensed national information infrastructure band and in the $2.4 \mathrm{GHz}$ ISM band. Both use orthogonal

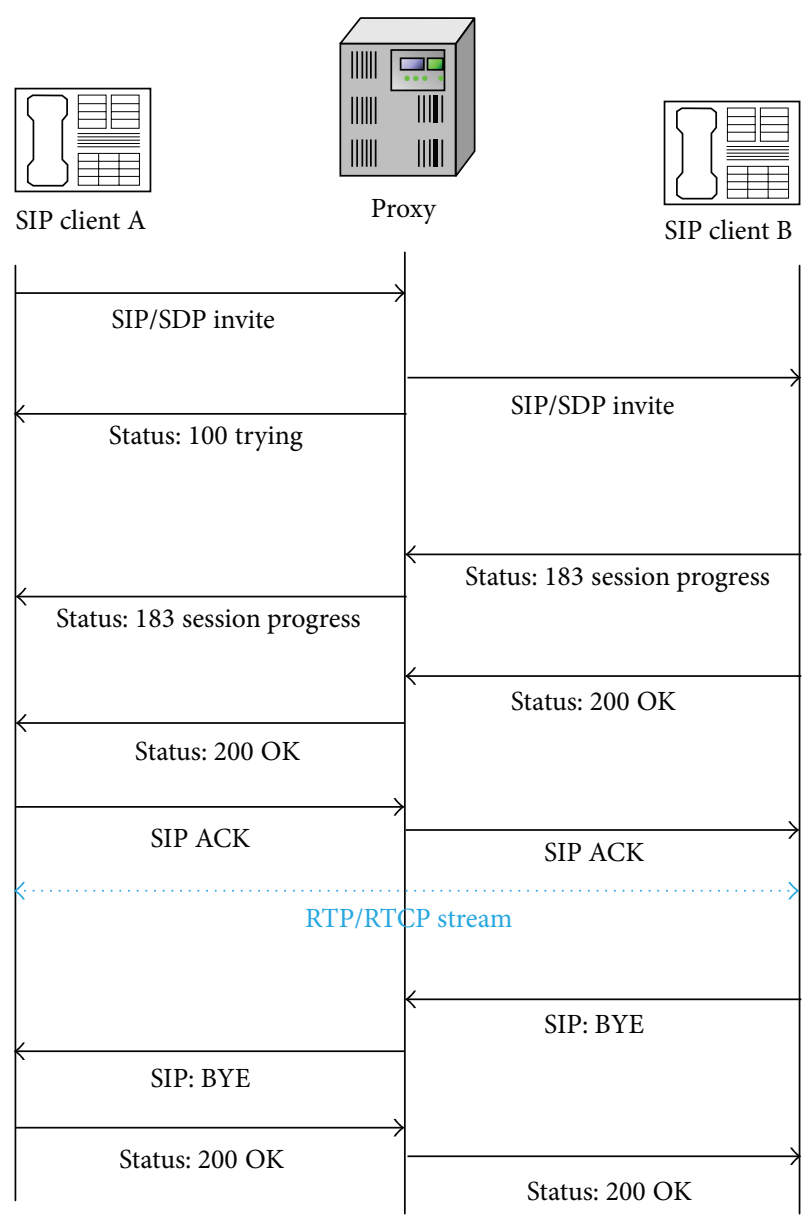

FIGURE 1: Call management by proxy.

frequency division multiplexing (OFDM) that divides the information into $N$ parallel streams which are transmitted by $N$ subcarriers. This increases the symbol duration by $N$ and the subcarriers are orthogonal so that the receiver can separate the signals carried by the subcarriers. 802.11a uses 48 subcarriers of the 64 subcarriers generated [1].

\section{SIP and H.323 Calls Management}

3.1. Proxy. Proxy is the main element of SIP infrastructure. Its role is to forward all signaling messages from transmitter to its eventual destination in order to establish and control connection between the call players.

Figure 1 shows different steps to establish a session between SIP client A and B by sending an invite message to the proxy. When the proxy is ready, it responds with a 100 trying message to inform that the message has been received.

Then proxy looks up URI of the second client in its contact list and makes sure that the destination is available to receive the forwarded message.

Client $\mathrm{B}$, in turn, acknowledges receipt of the message by sending the 180 session progress message. Proxy forwards this information to the call initiator to ensure that the line is available. So client B accepts the call and sends a 200 


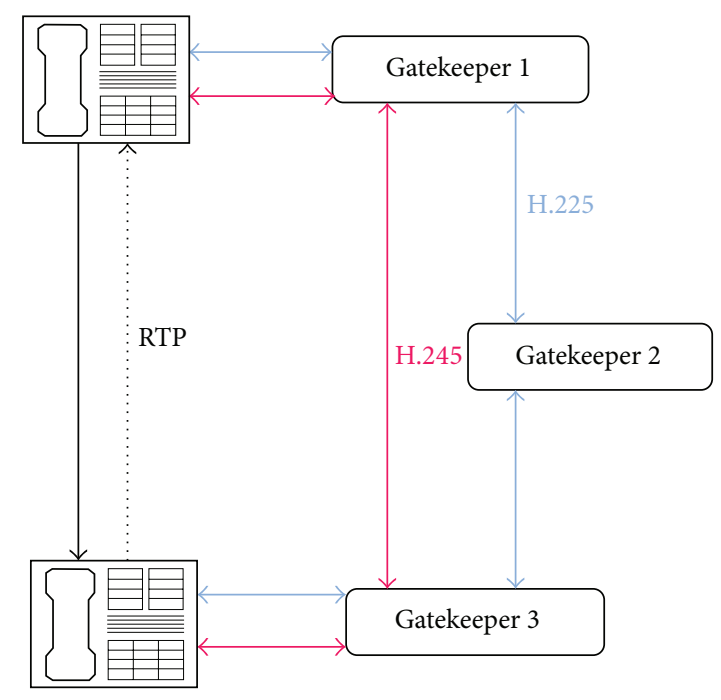

FIGURE 2: Diagram of a communication with 3 gatekeepers.

OK message; after that, proxy forwards it to client A. This initiator acknowledges that and proxy acknowledges receipt the message received by client $\mathrm{B}$.

After this session establishment, the two terminals communicate directly, peer to peer.

In the end of conversation, client $\mathrm{A}$ generates a bye message to finish the call and proxy, as done before, forwards that message and finally the user of the other machine sends $200 \mathrm{OK}$ which is forwarded by the proxy to the second user.

3.2. Gatekeeper. Gatekeepers perform a number of important functions that preserve the integrity of the corporate data network. The first one is address translation from H.323 aliases for terminals and gateways to network addresses, as defined in the RAS specification. The second function is access control. The third function is bandwidth management. For instance, if a network manager has specified a threshold for the number of simultaneous conferences on the LAN, the gatekeeper can refuse to make any more connections once the threshold is reached. The effect is to limit the total conferencing bandwidth; the remaining capacity is for email, file transfers, and other LAN activities.

The fourth function is to manage a number of terminals, gateways, and MCUs as a single logical group known as the H.323 zone $[7,8]$.

Figure 2 shows that to manage VoIP communications under H.323 protocol, gatekeepers need a number of subprotocols to establish successful calls between users.

3.3. OPNET Modeler Capabilities. Opnet Modeler is a software which supports the modeling of communication networks and distributed systems. This developed tool can analyze behavior and performance of simulated scenarios. The Opnet environment amalgamates different skills to succeed in all phases of the study (model design, simulation, data collection, and data analysis).

\section{Model for Performance Analysis of SIP and H.323 in Wireless Networks}

4.1. Motivation of Wireless SIP/H.323 Integration. In the previous years, several studies were conducted to analyze wireless VoIP technologies in order to explain its utility and develop other applications in this regard. That's why, nowadays, there is a number of soft applications and hard materials working on success of wireless VoIP communication using signaling protocols such as SIP and H.323.

However, to coexist in a same network wireless topology, all VoIP users must work under one signaling protocol. In this paper, using OPNET Modeler makes it possible to manage multiprotocol terminals (SIP and H.323) in one wireless network topology by developing a new wireless SIP Proxy prototype supporting H.323 protocol; it means that this new OPNET node will be able to be a wireless proxy and gatekeeper in the same time. When a SIP or H.323 user initiates a call, it sends a request to the proxy/gatekeeper recently created by specifying the destination address. So, the new device differentiates the type of message received by checking the format of callee and caller addresses and also by searching in the SIP and H.323 database and then the device can continue to establish normally the call.

4.2. Node Model. Node models are developed in the node editor and expressed in terms of smaller building blocks called modules. Some modules offer capability that is substantially predefined and can only be configured through a set of built-in parameters. These include various transmitters and receivers allowing a node to be attached to communication links in the network domain. Other modules, called processors and queues, are highly programmable, with their behavior being prescribed by an assigned process model [9].

As shown in Figure 3, the new node model of the proxy prototype recently created contain a number of modules which are the basic building blocks of that model which offers 


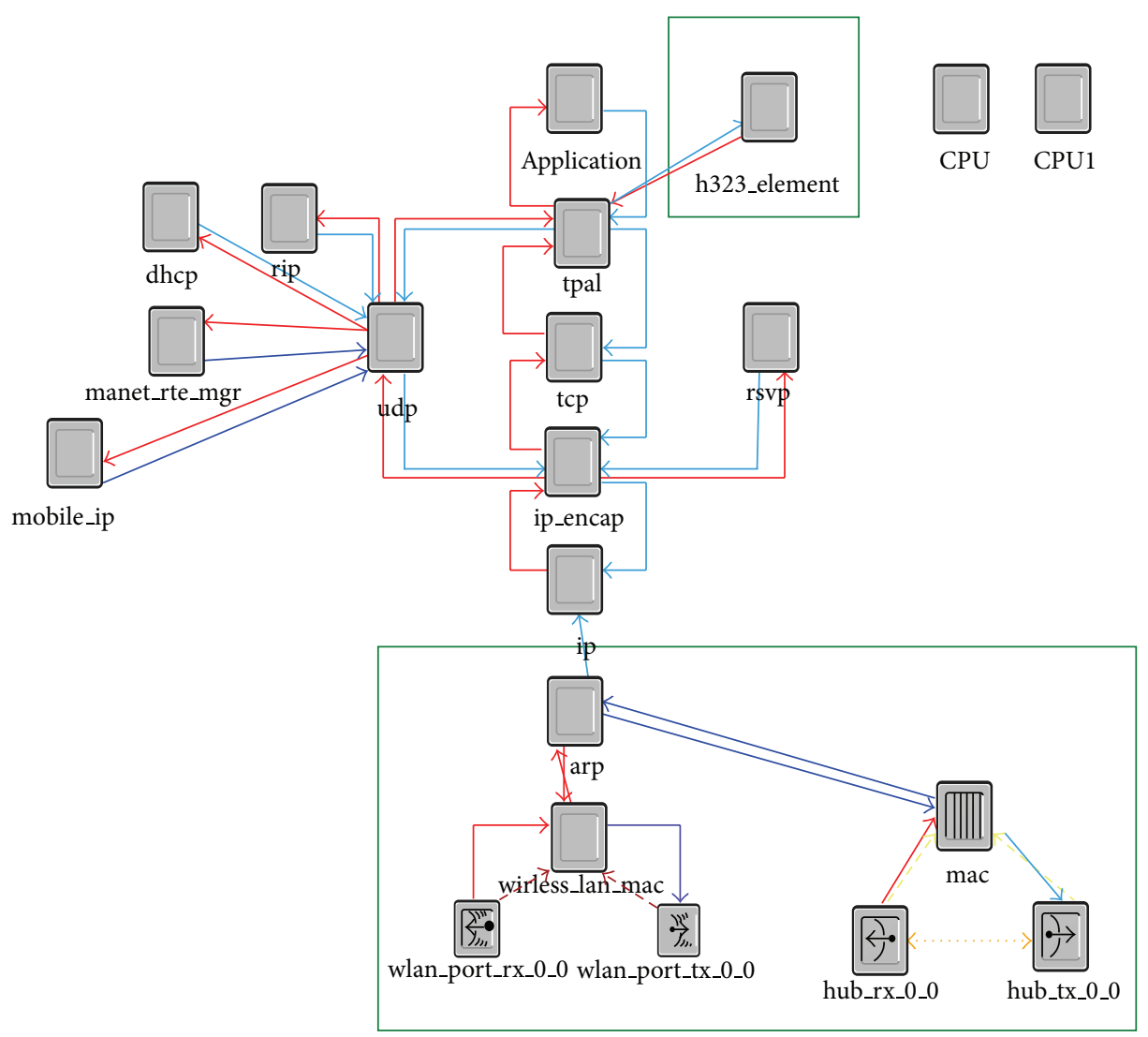

FIGURE 3: The node model of new created device.

all the functionality of processor and can also buffer and manage collection of data packets.

As mentioned earlier, that node model is a SIP Proxy model within new blocks; "h323_element" was added to make the management of H.323 and SIP calls in a same established scenario possible; "Mac" and "wireless LAN Mac" make it possible to make connectivity with both wired and wireless networks.

The "h323_element", "Mac", and "wireless LAN Mac" evidently contain a subnodes called process model which illustrates the compilation of an algorithm translated in $\mathrm{C}$ language.

\section{Discussion of the Results}

5.1. Operational New Wireless Proxy-Gatekeeper. According to the sections below, it is now possible, like never before, to create a complex wireless scenario containing both signaling protocols SIP and H.323 and evaluate their performances together in the same network. Two scenarios are done to conduct a comparison study of the two cases (wireless network within 3 access points 3 proxy-gatekeepers and 14 VoIP users versus the same composition of that network but with closer users) and show that the integration is successful in the first scenario as shown in Figure 4.

5.2. QoS Parameters. In this section, a comparison between all QoS parameters (jitter, MOS, end-to-end delay, sent, and received traffics) is done to show that the integration of both signaling protocol did not act negatively in the high-quality service offered by VoIP application but in the case of closer users from the access point, an important improvement of voice quality is noteworthy.

5.2.1. Jitter. The end-to-end delay variation between two consecutive packets is called jitter. A jitter of less than $50 \mathrm{~ms}$ is acceptable for high quality VoIP calls. If the delay of transmissions varies too widely in a VoIP call, the call quality is greatly degraded [10].

As shown in Figure 5, in the two cases (15 users and 15 closer users) the importance of jitter variation is very different; distance between users is a strong factor of increasing jitter. But we can say that in the two cases, the new device keeps in norms the jitter value less than $50 \mathrm{~ms}$.

5.2.2. MOS. The calculated MOS is a standard ETSI and ITUT. A mathematical calculation of this value could be realized by considering the characteristics of the communication.

This recommendation is based on the fact that the damage is added together on a scale of predetermined quality. If a signal passes through multiple devices, equipment damage adds up [7].

As shown in Figure 6, MOS value is more important and steady with closer users in spite of signaling integration (SIP and H.323). 


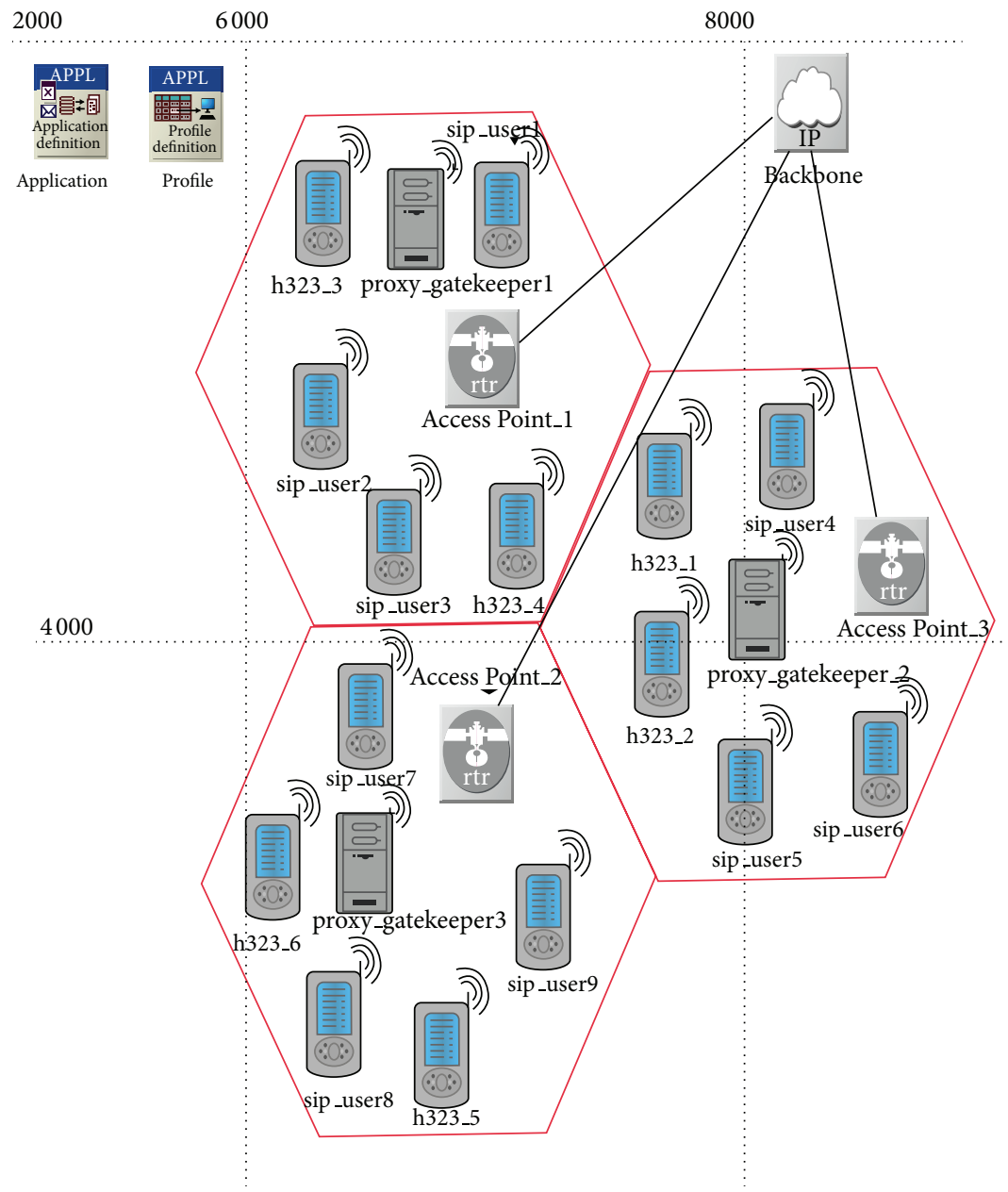

FIGURE 4: Wireless scenario with the new crated device.

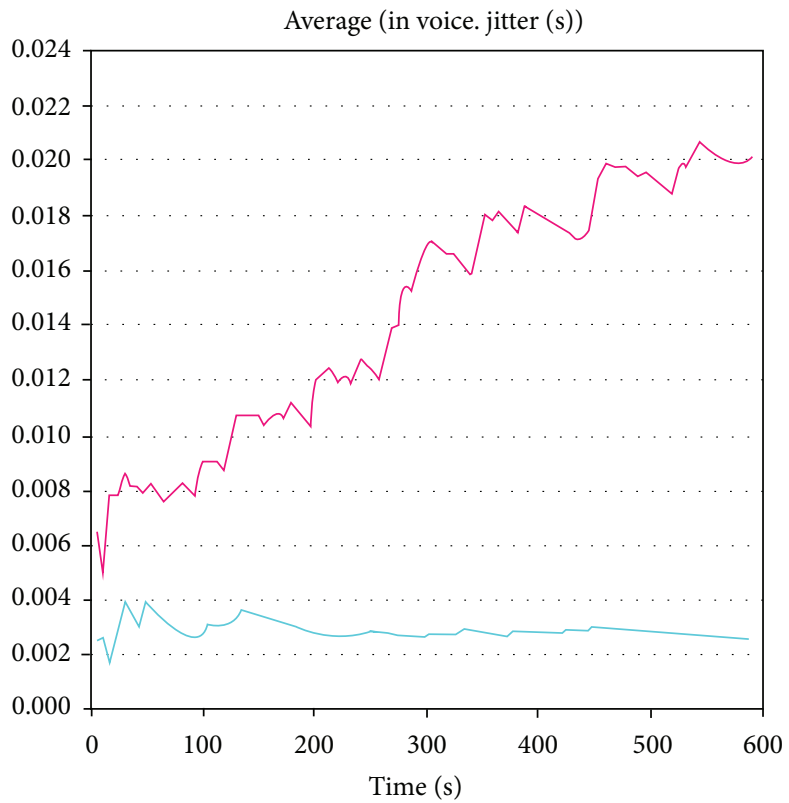

Voip_wifi-voip_closer_proxy_gatekeeper1-DES-1

_ Voip_wifi-voip-proxy-gatekeeper-DES-1

FIgURE 5: Voice jitter average. 


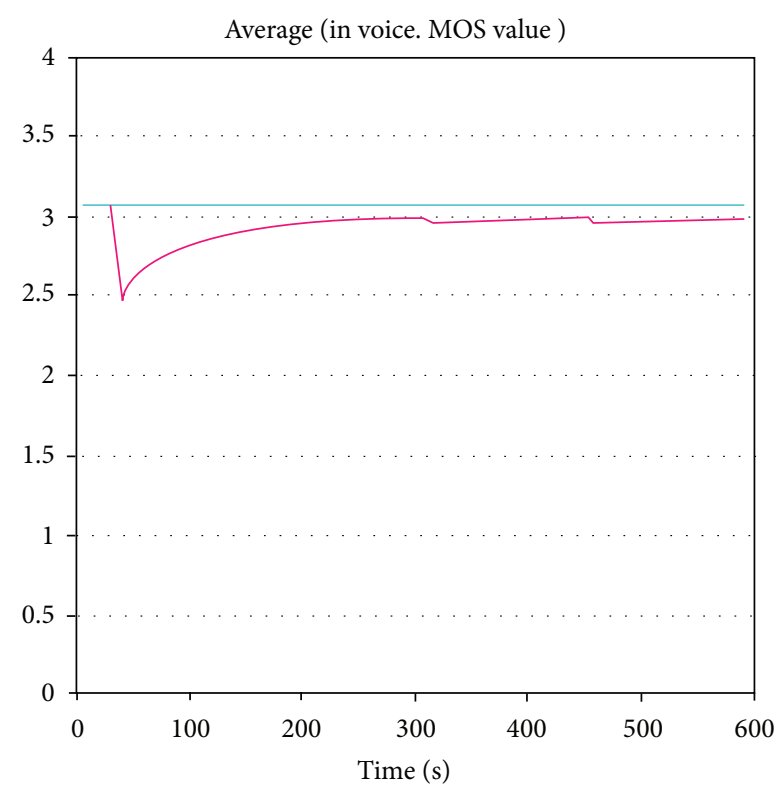

Voip_wifi-voip_closer_proxy_gatekeeper1-DES-1

_ Voip_wifi-voip-proxy-gatekeeper-DES-1

FIGURE 6: MOS average calculation.

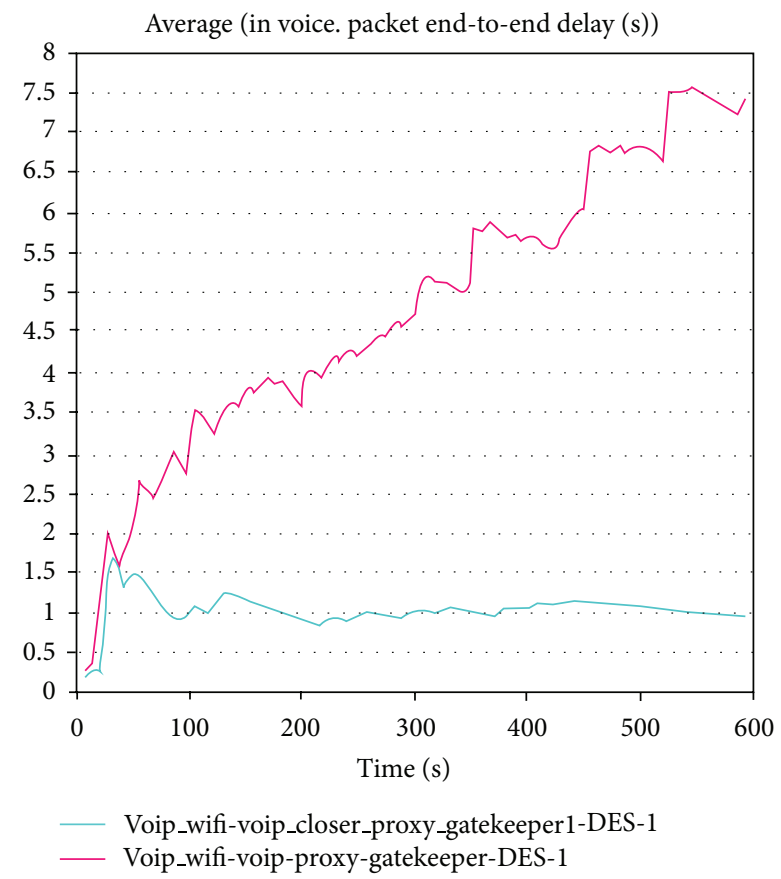

FIgURE 7: Packet end-to-end delay.

5.2.3. End-to-End Delay. End-to-end delay consists of endsystem and network delay. The end-system delay occurs due to the encoding and decoding delay and dejitter buffering delay [10].

Figure 7 shows that the end-to-end delay values are too advantageous in the closer users compared to the heterogeneous scenario with SIP/H.323 distant users.

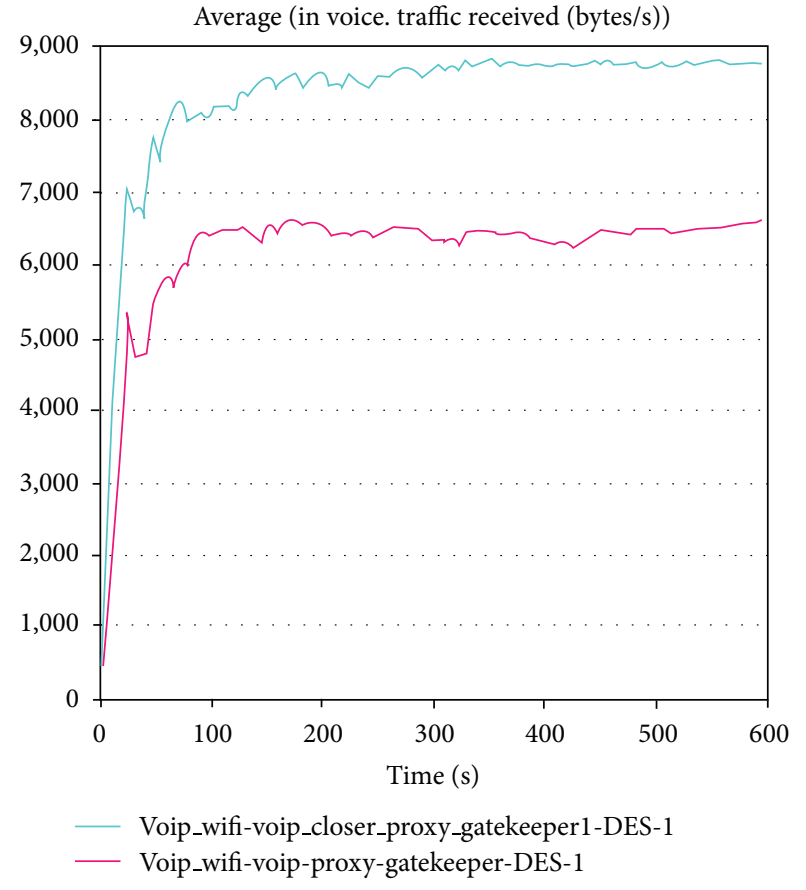

FIGURE 8: Received traffics.

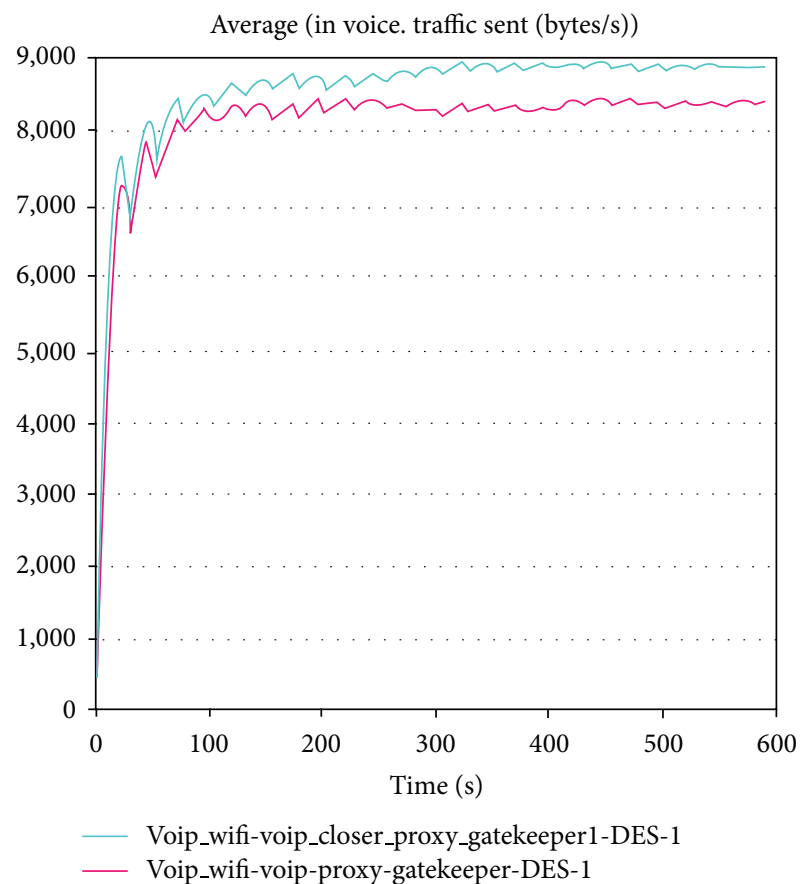

Figure 9: Sent traffics.

5.2.4. Sent/Received Packets. In this part of the comparison study, the sent and received traffics are shown in Figures 8 and 9 to explain that in both scenarios traffic sent has the same value but in the closer case, traffic received is more important, which means the fact that lower packet loss is obtained when the users are closer to the access point and to the new created device supporting the both signaling protocol SIP and H.323. 


\section{Conclusion}

This paper describes the steps followed to create a new wireless signaling device supporting both SIP and H.323 protocol (proxy/gatekeeper) to allow multiprotocol wireless network simulations in OPNET modeler which considered as a powerful tool of computer network analysis.

The creation of such tool allowed the construction of a wireless VoIP communication scenario, being controlled by two different signaling protocols in different ways. Consequently, this provides a high-quality service, comparing Jitter, end-to-end delay, MOS, received and sent traffic, and packet loss especially when wireless VoIP users working under the two protocols in the same time are so much closer to the proxy/gatekeeper and to the access point.

In short, this work has brought a multifunctionality of a basic unique wireless element, maintaining the better quality of the service.

In perspective, it is expected to add and create a new wireless ad hoc VoIP system where GPS helps users to find the closer proxy/gatekeeper to be connected in because according to this paper, we concluded that more users are closer QoS is better.

\section{Conflict of Interests}

The authors declare that there is no conflict of interests regarding the publication of this paper.

\section{Acknowledgement}

This work falls within the scope of telecommunication projects. The authors would like to thank the Department of technology of the MESFCRST for financing our projects.

\section{References}

[1] H. Fathi, S. S. Chakraborty, and R. Prasad, Voice over IP in Wireless Heterogenous Networks, Signaling, Mobility and Security, Springer, Amsterdam, The Netherlands, 2009.

[2] J. Rosenberg, H. Schulzrinne, G. Camarillo et al., "SIP: Session Initiation Protocol," Internet Engineering Task Force (IETF), RFC 3261, 2002.

[3] IEEE 802.11 WG. Part 11a/11b/11g, "Wireless LAN Medium Access Control (MAC) and Physical Layer (PHY) specifications," IEEE Standard Specification, 1999.

[4] F. A. Tobagi, "Multiaccess protocols in packet communication systems," IEEE Transactions on Communications, vol. 28, no. 4, pp. 468-488, 1980.

[5] T. Vo-Dai, "Throughput-delay analysis of the nonslotted and nonpersistent CSMA-CD protocol," in Local Computer Networks, P. C. Ravasio, N. G. Hopkins, and N. Naffah, Eds., pp. 459-476, North-Holland Publications, Amsterdam, The Netherlands, 1982.

[6] "Information Technology-Telecommunications and Information Exchange Between Systems-Local and Metropolitan Area Networks-Specific Requirements-Part 11: Wireless Lan Medium Access Control (MAC) and Physical Layer (PHY) Specifications," IEEE Std 802.11-1997, 1997.
[7] S. Rattal, A. Badri, and M. Moughit, "Performance analysis of hybrid codecs G.711 and G.729 over signaling protocols H.323 and SIP," International Journal of Computer Applications, vol. 72, pp. 30-33, 2013.

[8] ITU-T Recommendation H.323, Packet-based multimedia communications systems, November 2000.

[9] A. Chhabra and G. Singh, "Performance evaluation and delay modelling of VoIP traffic over 802.11 wireless mesh network," International Journal of Computer Applications, vol. 21, no. 9, pp. 7-13, 2011.

[10] A. Chhabra and G. Singh, "Performance evaluation and delay modelling of VoIP traffic over 802.11 wireless mesh network," International Journal of Computer Applications, vol. 21, no. 9, pp. 7-13, 2011. 

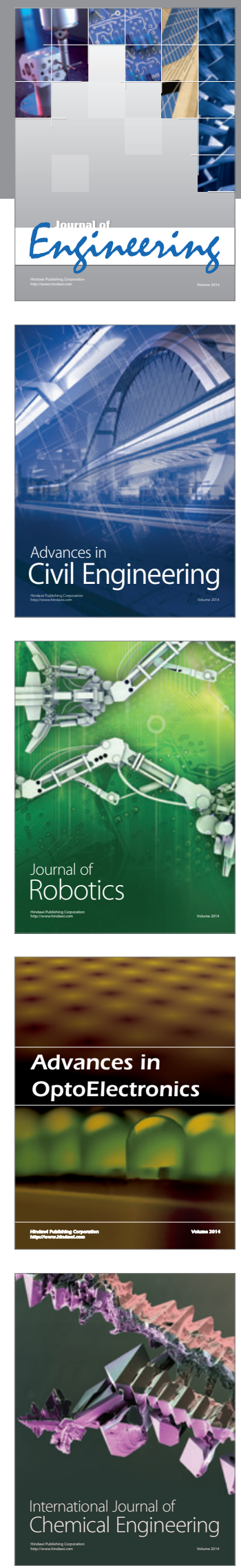

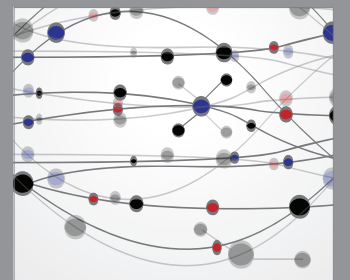

The Scientific World Journal
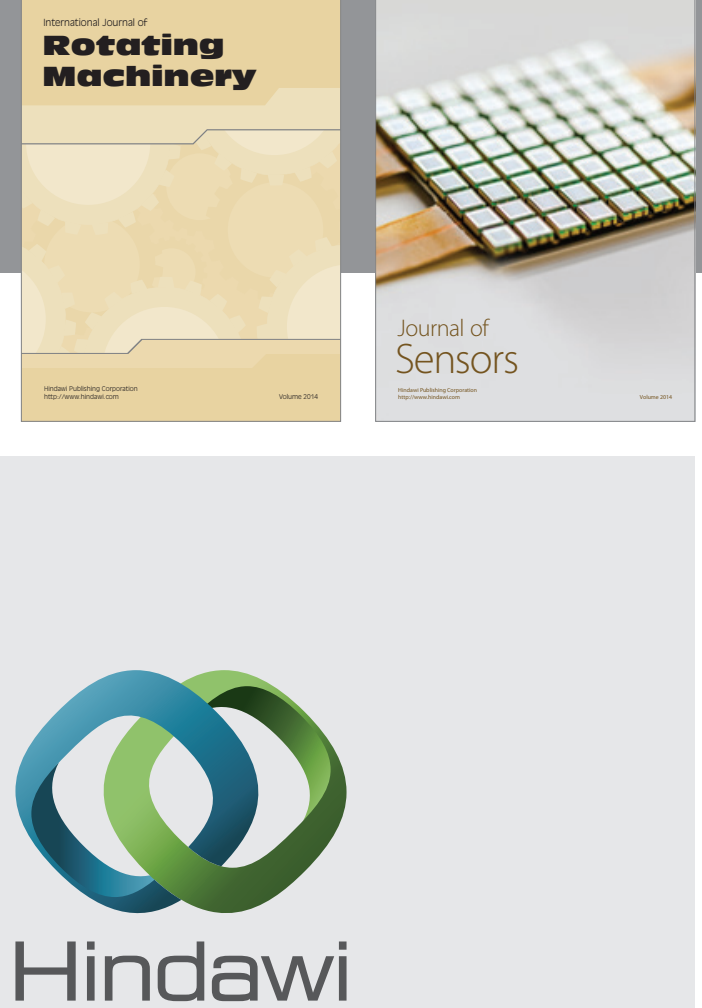

Submit your manuscripts at http://www.hindawi.com
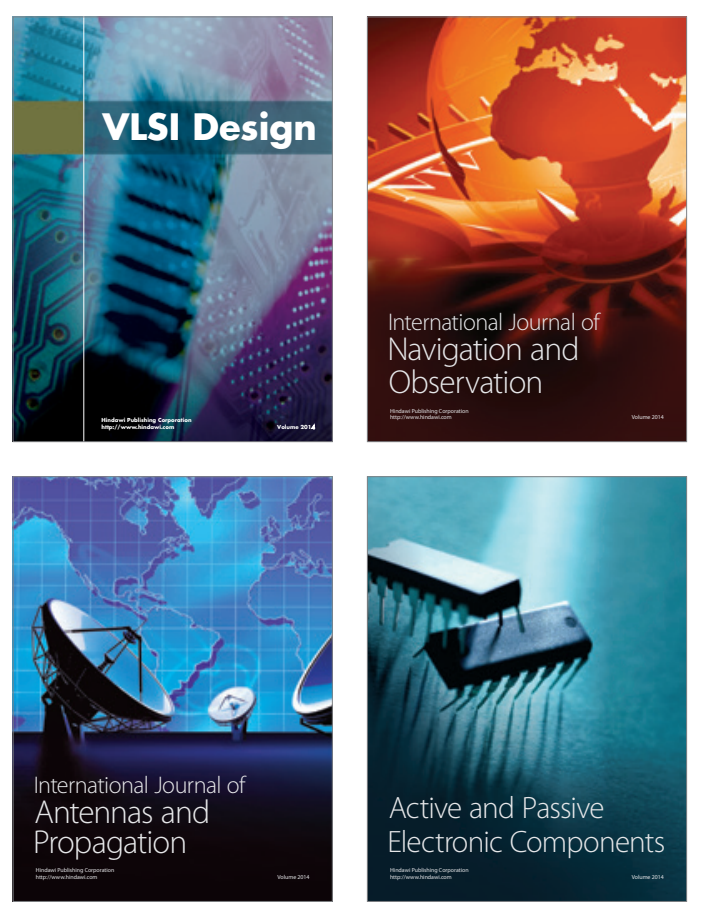
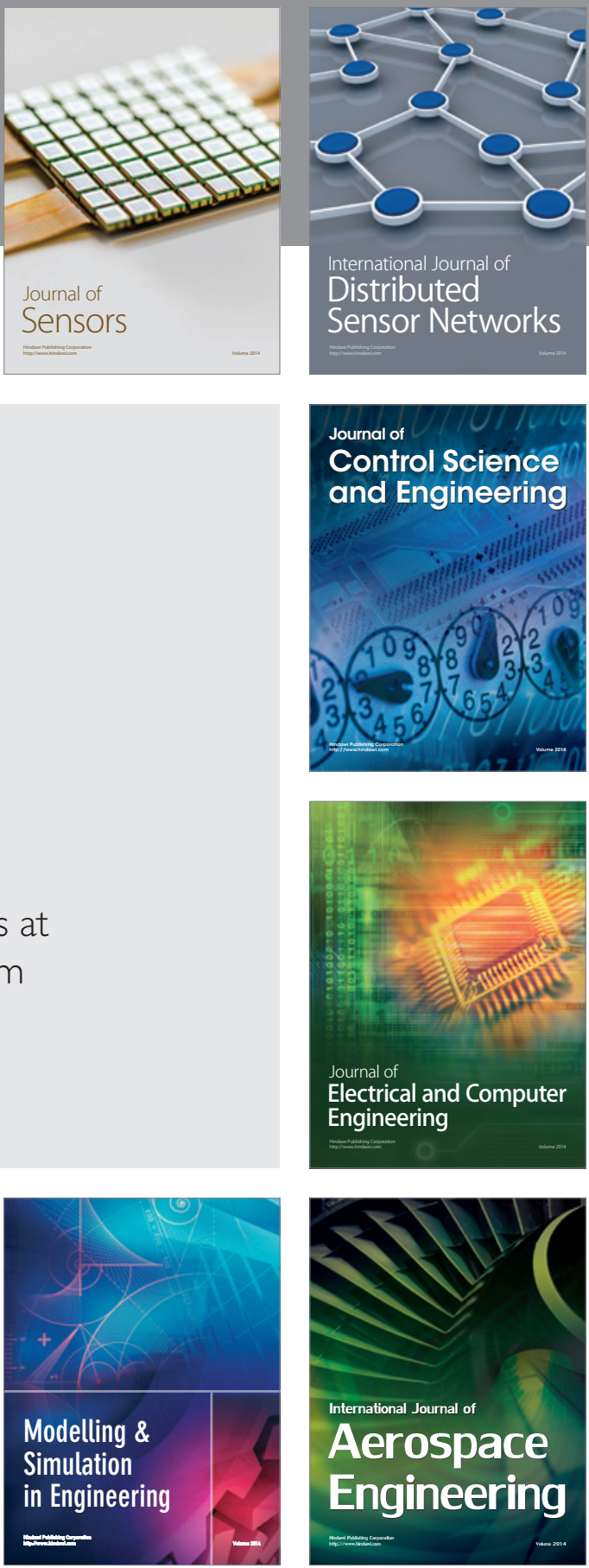

Journal of

Control Science

and Engineering
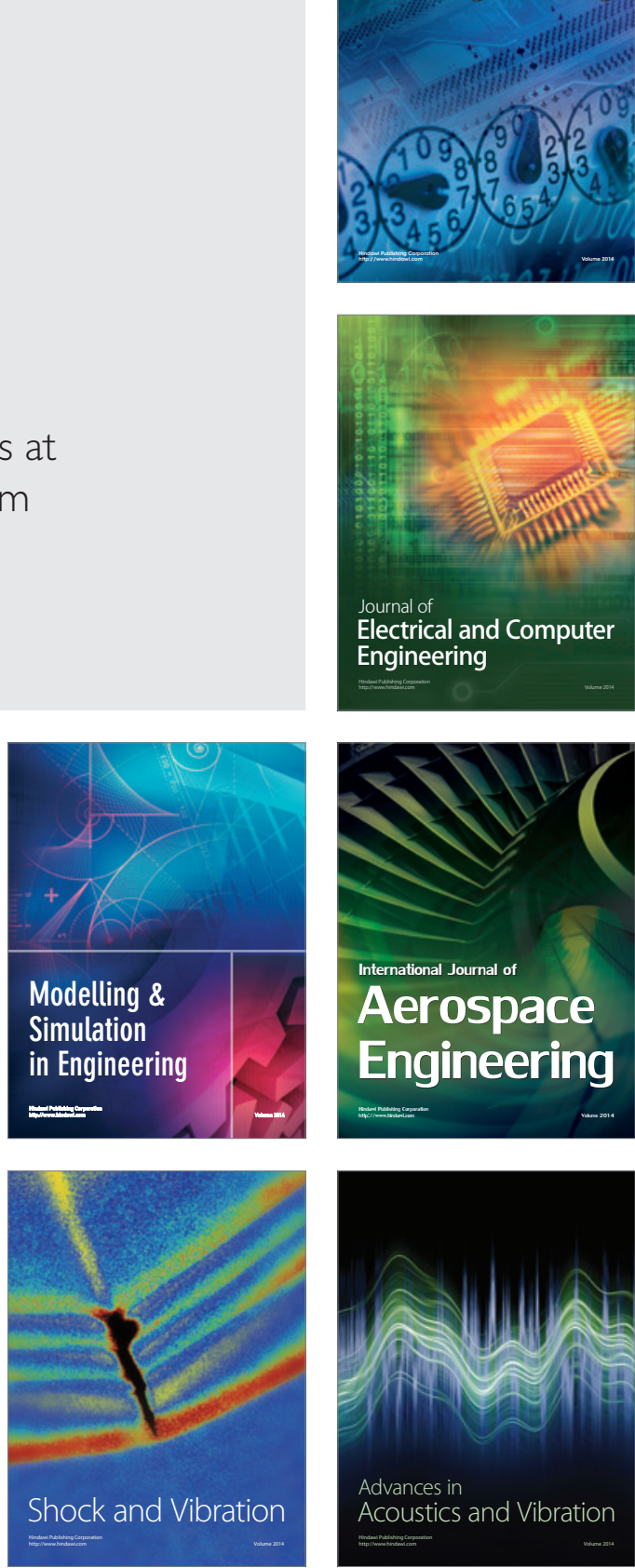